\title{
Mixed autoinflammatory and autoimmune syndrome
}

INSERM

\section{Source}

INSERM. (1999). Orphanet: an online rare disease and orphan drug data base. Mixed autoinflammatory and autoimmune syndrome. ORPHA:324933

Mixed autoinflammatory and autoimmune syndrome is a group of systemic diseases characterized by mixed patterns of dysregulated innate and/or adaptive immune responses, leading to chronic activation of the immune system and tissue inflammation, which presents clincially with a wide range of variable, concomitant, autoimmune and autoinflammatory manifestations in various organ systems. 